Research paper

\title{
Parametric study on drag reduction with the combination of the upstream energy deposition and the opposing jet configuration in supersonic flows
}

\author{
Shengjun $\mathrm{Ju}^{\mathrm{a}}$, Zhenxu Sun ${ }^{\mathrm{a}, *}$, Guowei Yang ${ }^{\mathrm{a}}$, Prasert Prapamonthon ${ }^{\mathrm{a}, \mathrm{b}}$, Junyuan Zhang ${ }^{\mathrm{c}}$ \\ ${ }^{a}$ Key Laboratory for Mechanics in Fluid Solid Coupling Systems Institute of Mechanics, Chinese Academy of Sciences, Beijing 100190, China \\ b International Academy of Aviation Industry, King Mongkut's Institute of Technology Ladkrabang, Bangkok 10520, Thailand \\ ${ }^{\mathrm{c}}$ School of Aerospace Engineering, Shenyang Aerospace University, Shenyang 110136, China
}

\section{A R T I C L E I N F O}

\section{Keywords:}

Computational fluid dynamics

Drag reduction

Supersonic flow

Energy deposition

Opposing jet

\begin{abstract}
A B S T R A C T
The drag reduction characteristics play an important role in the supersonic vehicle design phase. According to two drag reduction schemes, the opposing jet and the upstream energy deposition have aroused the widespread interest of researchers. In the current study, the drag reduction effectiveness of a blunt body with the combination of the upstream energy deposition and the opposing jet configuration in supersonic flows is investigated numerically. The three-dimensional coupled implicit compressible Reynolds Averaged Navier-Stokes (RANS) equations coupled with the Menter's shear stress transport (SST) turbulence model are applied to numerically predict flow fields of the blunt body, the variance analysis method is introduced to a parametric study on the drag reduction. Results indicate that a larger overall drag is decreased by the combinational configuration than the single strategies of the opposing jet and the energy deposition. The proposal of the combination of the upstream energy deposition and the opposing jet configuration can be used as an effective method of drag reduction. Meanwhile, due to the existence of the upstream energy deposition, the stability and penetrability of the opposing jet substantially increase, especially for the long penetration mode. Further, some recommendations are provided for the drag reduction factor and drag reduction effectiveness.
\end{abstract}

\section{Introduction}

In the supersonic flow field, the overall drag of a vehicle is dominated by the shock wave, which is generated at the leading edge. This can lead to a rapid increase in the aerodynamic drag significantly $[1,2]$. Because the shock wave structure can be obviously changed by the interaction between the opposing jet and the incoming flow [3], the opposing jet technology is an effective flow control approach to reduce the drag of the blunt body at supersonic speeds [4,5].

Several experimental and numerical studies have highlighted a major role of the opposing jet in drag reduction.

A series of systematic experiments on the jet flow from the blunt body opposing a supersonic free stream was carried out by Finley [6] An analytical model to investigate the flow field around various blunt shapes with an opposing jet was established. The result showed that the surface pressure and drag decreased by a wide margin with the opposing jet to the flow field.

The numerical simulation on drag reduction of a hemisphere model with an opposing jet in a supersonic flow was conducted by Hayashi [7]. It was found that two motion modes, separated by a critical point of jet-to-freestream total-pressure ratio $(P R)$, existed in the flow structures of the blunt body with the opposing jet, which included the long penetration mode (LPM) and the short penetration mode (SPM). The instability and oscillation of the jet flow field obviously appeared when the jet-to-freestream total-pressure ratio was small.

The large-eddy simulation (LES) was used by Chen [8] to numerically investigate the fundamental mechanisms of shock/opposing jet interaction, shock/shear-layer interaction, turbulent shear-layer evolution, and coherent structures in the opposing jet flow. The result showed that for the unstable long penetration state, the X-shaped shock structure was generated by the interaction of oblique shock and reflection shock. In addition, the Mach disk was established between the bow shock and opposing jet nozzle for the stable short penetration state.

In order to improve the drag reduction performance, many control techniques, for example, the aerospike [9-11] and the forward-facing cavity [12-15] combined with opposing jet were proposed to reduce the drag performance. The progress of the experimental and numerical investigation on the drag reduction mechanism induced by an opposing jet and its combinations has been summarized in detail $[16,17]$. The

\footnotetext{
* Corresponding author.

E-mail address: sunzhenxu@imech.ac.cn (Z. Sun).
} 
combinatorial configurations showed better drag reduction performance than for a single opposing jet, and more attention should be paid to combinations of the opposing jet and other techniques $[18,19]$.

The combinational novel cavity and opposing jet concept were proposed and the comparison of the drag and heat reduction efficiency of the conventional and parabolic cavity configurations were conducted by the numerical method [9]. It was found that the drag coefficient was increased by the control of the combined method. Moreover, the drag reduction efficiency of parabolic cavity configurations was higher than that of conventional cavity configurations at the low total pressure ratio.

The drag reduction mechanism of the combination of opposing jet and spike concept in supersonic flows was investigated numerically by Huang et al. [12]. The effects of aerospike length and jet pressure ratio were analyzed as well. The results showed that the combined method was more effective than the opposing jet only for drag reduction on the hypersonic blunt body. The results also indicated that the aerodynamic drag increased with the increase of the aerospike length and the jet pressure ratio.

In recent years, the combination of opposing jet and the upstream energy deposition has become interesting to researchers as a new combined flow control scenario because of its good effect on drag reduction and jet stability[20,21]. The combined method primarily has been studied and used to improve the performance of vehicles at supersonic/hypersonic speeds.

The concept of the combination of opposing jet and energy deposition to reduce the drag of the blunt body in hypersonic flow was first proposed by Khamooshi et al. [20]. The coupling effect of the opposing jet and upstream energy deposition of the blunt was studied experimentally and numerically. The results showed that the overall drag was greatly reduced by the combined strategy, whereas the stability of the opposing jet was significantly enhanced.

By means of numerical simulation, Marley et al. studied the jet stability combined with the annular ring injector as well as imposing swirl[21]. It was found that the energy deposition could push the bow shock wave away from the blunt body nose, which could effectively enhance the jet flow stability and penetration. Besides, the annular ring injection made the flow field more stable than swirled injection.

Based on the previous work on the combined method of the opposing jet and the upstream energy deposition, the critical parameters of the combined method for drag reduction can be identified by analyzing drag characteristics and jet flow stability of the blunt body. The understanding of the drag reduction mechanism on the combinational configuration of the upstream energy deposition and opposing jet can be further deepened.

In this study, the numerical simulation of the three-dimensional blunt body with the combination of the upstream energy deposition and opposing jet configuration in supersonic flows is performed to demonstrate and illuminate the characters and alterations of the flow field. Also, this study further investigates the effects of the flow control parameters on the drag reduction performance using the variance analysis method. The results of the variance analysis are verified and discussed.

\section{Physical model and numerical method application}

\subsection{Physical model}

In this study, the geometric model of a three-dimensional blunt body in supersonic flow is adopted from the open literature [7]. It consists of two parts i.e. hemispherical-nosed forebody and cylindrical main body. Fig. 1 shows the schematic diagram of the physical model employed in the current study. The radius of the hemispherical forebody $R_{\mathrm{b}}$ is $50 \mathrm{~mm}$, the main body is in a cylindrical shape with the same radius of $50 \mathrm{~mm}$, the length of the cylindrical main body $L_{\mathrm{b}}$ is $50 \mathrm{~mm}$, and the diameter of the opposing jet orifice $D_{\text {jet }}$ is $40 \mathrm{~mm}$. The previous

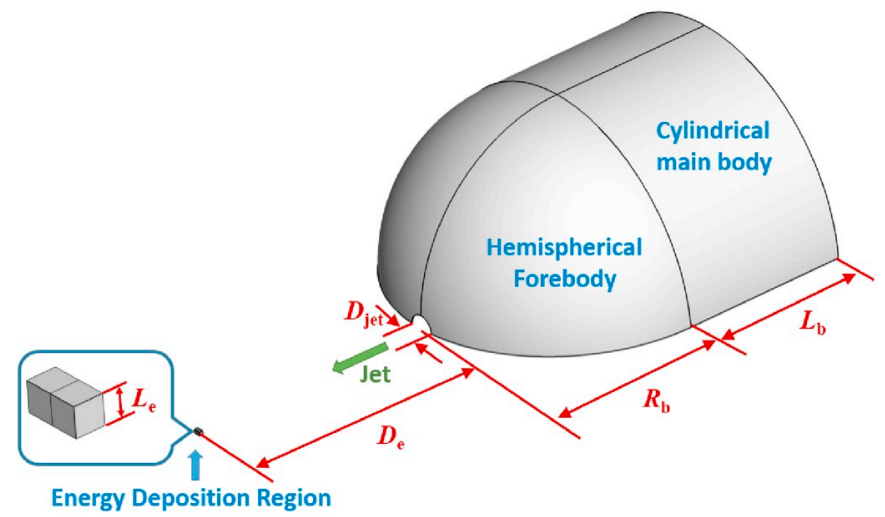

Fig. 1. Schematic diagram of the geometrical models.

studies suggested that both the location of energy deposition and the energy deposition power had a significant impact on the drag reduction [22]. For the quarter physical model, the energy deposition is considered as uniform cubes and the side length of energy deposition cube $L_{\mathrm{e}}$ is $0.50 \mathrm{~mm}$. The density of the energy deposition $S_{\mathrm{e}}$ is $260 \mathrm{~W} \mathrm{~mm} \mathrm{~m}^{-3}$. It may be noted that ' $S_{\mathrm{e}}$ ' is the amount of energy added per unit volume $\left(Q / L_{\mathrm{e}}{ }^{3}\right)$ in W.mm ${ }^{-3}$, where, $Q$ is the energy deposition power and $L_{\mathrm{e}}{ }^{3}$ is the volume of cubic energy spot. The location of the energy deposition is on the upstream of the stagnation point and the distance between the nose of the blunt body and the energy spot $D_{\mathrm{e}}$ is $37.50 \mathrm{~mm}$.

Air is assumed as perfect gas and the Mach number of supersonic freestream flows $M a_{\infty}$ is set to be 3.98 assumed to be perfect gas and the total temperature $T_{\infty}$ and total pressure $P_{\infty}$ of the freestream are $397 \mathrm{~K}$ and 1.37 MPa, respectively. The opposing jet fluid is compressed air and its Mach number $M a_{\mathrm{j}}$ is 1.0 . The jet total temperature $T_{\mathrm{j}}$ is set as $300 \mathrm{~K}$ and the jet-to-freestream total-pressure ratio $P R$ is $0.2,0.4$, and 0.6 , respectively. Table 1 provides the corresponding flow conditions.

\subsection{Governing equations and turbulence model}

The three-dimensional steady RANS equations [23,24] are solved numerically to calculate the aerodynamics of the blunt body. The governing equations are described as follow:

Continuity equation:

$\frac{\partial \rho}{\partial t}+\frac{\partial \rho u_{i}}{\partial x_{i}}=0$

Momentum equation:

$\frac{\partial\left(\rho u_{i}\right)}{\partial t}+\frac{\partial\left(\rho u_{i} u_{j}\right)}{\partial x_{j}}=-\frac{\partial p}{\partial x_{i}}+\frac{\partial \tau_{i j}}{\partial x_{j}}$

Energy equation:

$\frac{\partial(\rho E)}{\partial t}+\frac{\partial\left(\rho u_{j} H\right)}{\partial x_{j}}=\frac{\partial\left(u_{i} \tau_{i j}\right)}{\partial x_{j}}-\frac{\partial}{\partial x_{j}}\left(\left(\frac{\mu_{L}}{\operatorname{Pr}_{L}}+\frac{\mu_{T}}{\operatorname{Pr}_{T}}\right) \frac{\partial T}{\partial x_{j}}\right)+S_{\mathrm{e}}$

where $\rho$ is the density, $u_{i}$ is the $i$ th component of the velocity, $H$ is the total enthalpy, $p$ is the pressure, $E$ is the total energy, $T$ is the temperature, and $\tau_{i j}$ is the shear stress tensor. The laminar Prandtl number

Table 1

Flow conditions.

\begin{tabular}{lll}
\hline Freestream & Gas & Air \\
& Mach number & 3.98 \\
& Total pressure & $1.37 \mathrm{MPa}$ \\
Opposing jet & Total temperature & $397 \mathrm{~K}$ \\
& Gas & Air \\
& Mach number & 1.0 \\
& Jet-to-freestream total-pressure ratio, PR & $0.2,0.4,0.6$ \\
& Total temperature & $300 \mathrm{~K}$
\end{tabular}


$\operatorname{Pr}_{L}$ and turbulent Prandtl number $\operatorname{Pr}_{T}$ are assumed to be 0.72 and 0.9 respectively. The laminar dynamics viscosity coefficient $\mu_{L}$ and turbulent viscosity coefficient $\mu_{T}$ are calculated by Sutherland law and RANS turbulence model respectively [23,24].

Previous studies have shown that the heating effect can be reasonably approximated via an energy addition rate with uniform distribution. The present simulations employ the uniform distribution defined in Eq. (4). Here, $Q$ describes the energy deposition power and $V_{\mathrm{e}}$ is the volume of cubic energy spot. $S_{\mathrm{e}}$ stands for the source term of the energy deposition (also known as the density of the energy deposition), which is set to be 0 when the case without the energy deposition is computed $[25,26]$.

$S_{\mathrm{e}}=\frac{Q}{V_{\mathrm{e}}}$

The pressure $p$ is acquired from the state equation for perfect gas:

$p=\rho R T=(\gamma-1)\left[\rho E-\frac{1}{2} \rho\left(u^{2}+v^{2}+w^{2}\right)\right]$

$E=e+\frac{1}{2}\left(u^{2}+v^{2}+w^{2}\right) H=e+\frac{p}{\rho}$

where $u, v$, and $w$ are the velocity in $\mathrm{x}-, \mathrm{y}-$, and z-axis of the Cartesian coordinates, respectively. $e$ is the internal energy, and $\gamma$ denotes the specific heat ratio. The shear stress term $\tau_{\mathrm{ij}}$ is the sum of a laminar and a turbulent component. The laminar viscosity is calculated by Sutherland formula, whereas the turbulent viscosity is calculated by SST $k-\omega$ turbulence model.

The turbulence model has a great impact on the predicted results in the numerical simulation $[27,28]$, the previous results show that the SST $k-\omega$ model is more suitable for flow field prediction of the counterflowing jet on a blunt body in supersonic flows [29,30]. The SST $k-\omega$ turbulence model was developed by Menter [31] with a significant influence from the original $k-\omega$ model of Wilcox and the standard $k-\varepsilon$ model. This model provides a low computational cost and robust calculations. It is widely used to simulate hypersonic flows in engineering applications. The model is composed of two-transport equations, namely.

Transport equation of turbulent kinetic energy $k$ :

$\frac{\partial(\rho k)}{\partial t}+\frac{\partial\left(\rho u_{j} k\right)}{\partial x_{j}}=\frac{\partial}{\partial x_{j}}\left[\left(\mu_{L}+\sigma_{k} \mu_{T}\right) \frac{\partial k}{\partial x_{j}}\right]+P_{k}-\beta^{*} \rho \omega k$

Transport equation of the specific dissipation rate of turbulence $\omega$ :

$$
\begin{aligned}
\frac{\partial(\rho \omega)}{\partial t}+\frac{\partial\left(\rho u_{j} \omega\right)}{\partial x_{j}}= & \frac{\partial}{\partial x_{j}}\left[\left(\mu_{L}+\sigma_{\omega} \mu_{T}\right) \frac{\partial \omega}{\partial x_{j}}\right]+P_{\omega}-\beta \rho \omega^{2} \\
& +2\left(1-f_{1}\right) \frac{\rho \sigma_{\omega 2}}{\omega} \frac{\partial k}{\partial x_{j}} \frac{\partial \omega}{\partial x_{j}}
\end{aligned}
$$

where $\mu_{L}$ and $\mu_{T}$ are the laminar and turbulent viscosity, respectively, and $P_{k}$ and $P_{\omega}$ denote production terms of the turbulent kinetic energy $k$ and the specific dissipation rate of turbulence $\omega$, respectively, and they are defined as [31].

$P_{k}=\mu_{T} \Omega^{2} P_{\omega}=C_{\omega} \rho \Omega^{2}$

where $\Omega$ is the magnitude of vorticity.

The turbulent viscosity is given by

$\mu_{T}=\frac{a_{1} \rho k}{\max \left(a_{1} \omega, f_{2}\|\Omega\|\right)}$

The SST model utilizes the standard $k-\varepsilon$ model in the outer region and in free shear layers and switches to the original $k-\omega$ model in the boundary layer near the sold wall [31,32]. Thus, two sets of constants $\phi_{1}$ and $\phi_{2}$ are used in the equations above and given as follow:

$\varphi=f_{1} \varphi_{1}+\left(1-f_{1}\right) \varphi_{2} f_{1}=\tanh \left(\Gamma_{1}^{4}\right)$

$$
\begin{aligned}
\Gamma_{1}= & \min \left[\max \left(\frac{\sqrt{K}}{0.09 \omega d}, \frac{500 \mu_{L}}{\rho \omega d^{2}}\right), \frac{4 \rho \sigma_{\omega 2} K}{C D_{K \omega} d^{2}}\right] C D_{K \omega}=\max \\
& \left(2 \frac{\rho \sigma_{\omega 2}}{\omega} \frac{\partial K}{\partial x_{j}} \frac{\partial \omega}{\partial x_{j}}, 1 \times 10^{-20}\right)
\end{aligned}
$$

where $d$ is the distance to the nearest wall, the constants $K=0.41$, $a_{1}=0.31$, and the $f_{2}$ term is given by:

$f_{2}=\tanh \left(\Gamma_{2}^{4}\right) \quad \Gamma_{2}=\max \left(\frac{2 \sqrt{K}}{0.09 \omega d}, \frac{500 \mu_{L}}{\rho \omega d^{2}}\right)$

The constants of $\sigma_{k}, \sigma_{\omega}, \beta$ and $\mathrm{C}_{\omega}$ for set 1 and set 2 are

$\sigma_{k 1}=0.85, \sigma_{\omega 1}=0.5, \beta_{1}=0.075, C_{\omega 1}=0.533$

$\sigma_{k 2}=1.0, \sigma_{\omega 2}=0.856, \beta_{2}=0.0828, C_{\omega 2}=0.440$

\subsection{Discretization and boundary conditions}

The governing equations discussed above are discretized by the finite volume approach based on the multi-block structured meshes. Inviscid fluxes are discretized by Roe flux-difference scheme with second-order Monotone Upstream-centered Schemes for Conservation Laws (MUSCL) reconstruction and minmod limiter [25]. The viscous fluxes are discretized by the second-order central difference scheme. The implicit Lower-Upper Symmetric Gauss-Seidel (LUSGS) scheme is employed for time advancement. The Courant-Friedrichs-Levy (CFL) number is kept at 0.75 with proper under-relaxation factors to ensure stability [26].

The solid wall condition, which assumes the non-slip for velocity, zero gradient of pressure and an isothermal wall temperature of $295 \mathrm{~K}$, is employed for the wall surface [32]. The velocity normal to the symmetry plane is zero. Besides, the freestream levels of $k, \omega$ and $\mu_{\mathrm{T}}$ at far-field boundaries for SST k-w turbulence model are specified as [31]:

$\frac{k_{\infty}}{a_{\infty}^{2}}=9 \times 10^{-9}, \frac{\omega_{\infty}}{\left(\rho_{\infty} a_{\infty}^{2}\right) / \mu_{\mathrm{L} \infty}}=1 \times 10^{-6}, \frac{\mu_{\mathrm{T}, \infty}}{\mu_{\mathrm{L}, \infty}}=0.009$

On the solid wall, the values of $k$ and $\omega$ are determined, respectively, as:

$k_{\text {wall }}=0, \quad \omega_{\text {wall }}=\frac{60 \mu_{1}}{0.075 \rho_{1}\left(d_{1}\right)^{2}}$

where $\mu_{1}$ and $\rho_{1}$ are the laminar viscosity and density, respectively. $d_{1}$ is the distance from the cell centers of first grids near the solid wall to the solid wall [32].

\subsection{Grid sensitivity analysis}

The grid sensitivity analysis of the opposing jet configuration is considered since the first cell height of the solid wall in the normal direction is very important for the numerical simulation, which is controlled by grid Reynolds number $R e_{\text {grid }}$ [33].

$R e_{\text {gird }}=\frac{\rho_{\infty} u_{\infty} d}{\mu_{\infty}}$

where $\rho_{\infty}, u_{\infty}$, and $\mu_{\infty}$ denote the density, velocity, and viscosity of freestream, respectively. $d$ is the first grid height in the direction normal to solid walls.

The jet-to-freestream total-pressure ratio $P R$ is set as 0.4 to ensure steady-state opposing jet flow fields. Three numbers of the computational grid of the model without the energy deposition are utilized to perform the grid sensitivity, namely, coarse grid, medium grid, and fine grid. Details of the three numbers of the grid are tabulated in Table 2 and the medium grid is presented in Fig. 2.

The comparison of the surface pressure distributions of the three numbers of the grid is presented in Fig. 3. It can be noticed that the 
Table 2

Grid system information.

\begin{tabular}{llll}
\hline & Coarse grid & Medium grid & Fine grid \\
\hline First cell height, mm & 0.0017 & 0.0011 & 0.0006 \\
$R e_{\text {grid }}$ & 67.8 & 45.2 & 22.6 \\
Cell number & $4.25 \mathrm{E}+06$ & $6.01 \mathrm{E}+06$ & $7.69 \mathrm{E}+06$
\end{tabular}

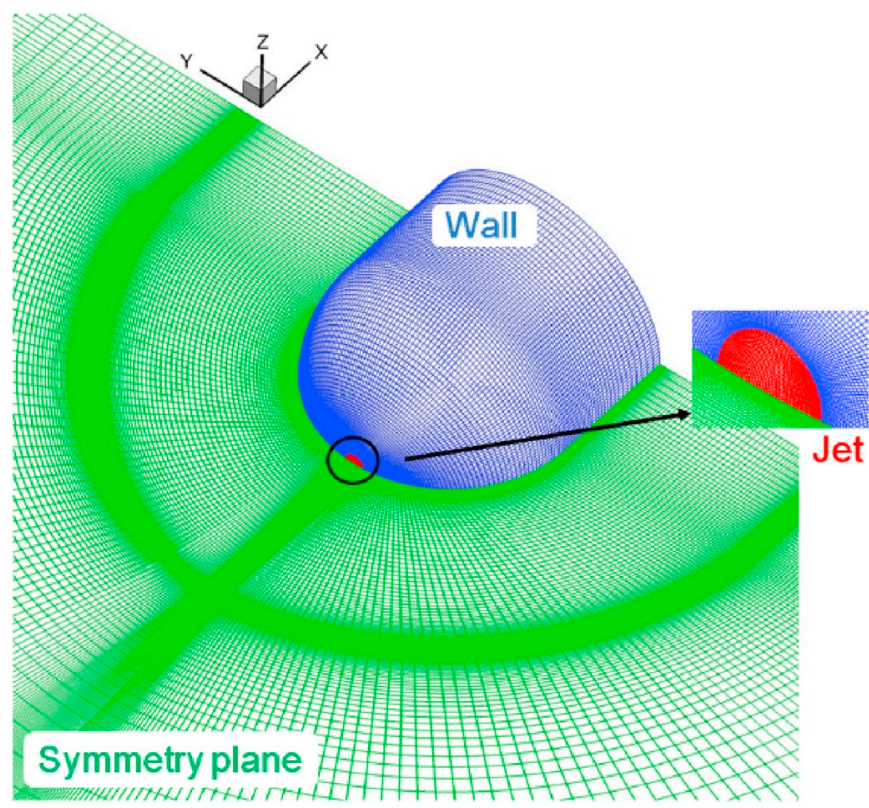

Fig. 2. Structured meshes of the blunt body.

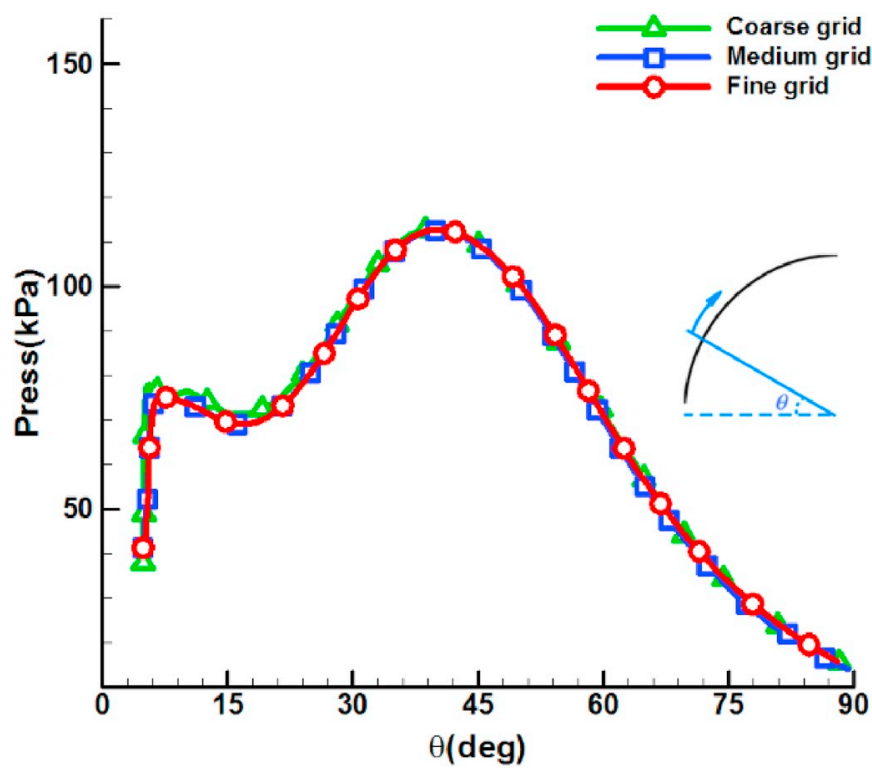

Fig. 3. Pressure comparison of three grid scales.

results of three different grid scales have slight differences and the magnitude and location of the peak pressure are highly consistent. Therefore, to save the computational cost and time-consuming, the medium-grid scale is used to guarantee the numerical precision for the following simulations.

\subsection{Accumulation of errors}

Estimating precision and error accumulation is necessary for numerical simulations in steady-state flows. The governing equations are discretized and solved on finite grid size. Therefore, a definite error occurs in integration at each step and the accumulation of numerical error is proportional to the number of integration steps [34,35]. The maximal allowable steps is introduced as an important characteristic for estimating precision and errors accumulation, The maximal allowable number of integration steps could be determined by the following formula $[34,35]$ :

$n_{\max }=\left(\frac{S^{\max }}{S_{\text {err }}}\right)^{2} \quad S_{\text {err }}=\sum S_{i}$

where $S^{\max }$ is the allowable value of total error and it is presumed to be between $1 \%$ and $5 \% . S_{i}$ and $S_{\text {err }}$ denote the relative error of integration in the " $i$ " direction and all-dimensional cases, respectively. The $S_{i}$ is defined by Ref. [34,35]:

$S_{i} \approx\left(\frac{\Delta L}{L_{i}}\right)^{k+1}$

where $\Delta L$ and $L_{i}$ denote the mean ratio of cell size and the size in the " $i$ " direction, $k$ is the order of accuracy of the numerical scheme. For a uniform grid, it could be $S_{i} \approx\left(1 / N_{i}\right)^{k+1}$, where $N_{i}$ is the number of cells in the direction of integration. Two structured grid blocks are generated and second-order schemes are utilized.

The ratio of the maximal allowable number of integration steps $R_{\mathrm{s}}$ is defined by the maximal allowable number of integration steps $n_{\max }$ and the number of integration steps $n[34,35]$.

$R_{s}=\frac{n_{\max }}{n}$

In the present simulation, three domains structured meshes are generated and aerodynamics are converged by nearly 50,000 steps. Table 3 tabulates the information of error accumulation for the three grids. Obviously, all the values of $R_{\mathrm{s}}$ are larger than unit andthe accumulated error does not exceed the maximal allowable value.

\subsection{Numerical validation}

To validate the numerical approach and CFD code, the pressure and flow field structure distributions of the opposing jet on the blunt body are compared against the experimental data provided by Hayashi [7] and the visualization of flow around the blunt body is obtained by Schlieren method. In order to confirm a steady-state of the jet flow structure, $P R$ is set as 0.4 and 0.6. The flow conditions are given in Table 1.

As shown in Fig. 4, details of the distinct structure such as the bow shock, barrel shock, reattachment shock, and the Mach disk, can be captured by the predicted approach. It is also observed that the flow field structures of the calculated jet are accord with photographs of experimental results found by Schlieren [7]for all cases.

The centerline surface pressure distributions at the three pressure ratios are also used to validate the numerical approach, as shown in Fig. 5. It can be observed that the calculated results show good agreement with the reference-predicted values of Hayashi et al. [7]. Therefore, the present numerical method can be used because it provides acceptable results with high accuracy and reliability. The numerical approach is also expected to predict complex flow structures accurately.

\section{Results and discussion}

According to flow structures of the opposing jet of experiments and numerical simulations obtained by Hayashi [7], both of the long penetration mode (LPM) and the short penetration mode (SPM) are presented. The two motion modes are separated by a critical point of the $P R$. At $P R=0.20$, the unstable and self-induced LPM mode emerges with oscillating motions. As $P R$ rises, nearly steady motion of the SPM 
Table 3

Accumulate error for the three grids.

\begin{tabular}{|c|c|c|c|c|c|c|c|c|}
\hline & $S_{1}$ & $S_{2}$ & $S_{3}$ & $\mathrm{~S}_{\mathrm{err}}$ & $S^{\max }$ & $\mathrm{n}$ & $n_{\max }$ & $R_{\mathrm{s}}$ \\
\hline \multirow[t]{2}{*}{ Coarse grid } & 3.08E-08 & $1.63 \mathrm{E}-06$ & 4.45E-07 & $2.10 \mathrm{E}-06$ & 0.01 & 50,000 & $2.26 \mathrm{E}+07$ & 451.81 \\
\hline & 3.08E-08 & 2.79E-06 & $3.36 \mathrm{E}-05$ & 3.64E-05 & 0.01 & 50,000 & $7.55 E+04$ & 1.51 \\
\hline \multirow[t]{2}{*}{ Medium grid } & 2.73E-08 & $1.28 \mathrm{E}-06$ & $2.40 \mathrm{E}-07$ & $1.55 \mathrm{E}-06$ & 0.01 & 50,000 & $4.16 \mathrm{E}+07$ & 831.22 \\
\hline & 2.73E-08 & $1.88 \mathrm{E}-06$ & $1.45 \mathrm{E}-05$ & $1.64 \mathrm{E}-05$ & 0.01 & 50,000 & $3.71 \mathrm{E}+05$ & 7.42 \\
\hline \multirow[t]{2}{*}{ Fine grid } & $2.52 \mathrm{E}-08$ & $1.20 \mathrm{E}-06$ & $1.44 \mathrm{E}-07$ & 1.37E-06 & 0.01 & 50,000 & $5.31 \mathrm{E}+07$ & 1061.39 \\
\hline & $2.52 \mathrm{E}-08$ & $1.33 \mathrm{E}-06$ & 7.54E-06 & $8.89 \mathrm{E}-06$ & 0.01 & 50,000 & $1.27 \mathrm{E}+06$ & 25.30 \\
\hline
\end{tabular}

mode is observed at $P R=0.40$.

In order to analyze the drag reduction characteristics using an opposing jet coupled with an energized region of the upstream flow, the modification of the supersonic flow field around the blunt body is investigated. The solutions of the baseline (no flow field modification), the energy deposition alone and the opposing jet alone, as well as, the combination of the upstream energy deposition and opposing jet configuration are numerically simulated in this section. The flow conditions of the computations carried out are presented in Table 1.

\subsection{Comparison of the drag reduction characteristics}

When the drag reduction characteristics are discussed, the drag reduction factor $(R D)[36,37]$ should be taken into consideration. This factor is defined as the modified drag value divided by the baseline (no flow field modification or injection) drag.

$R D=\frac{D_{\text {ref }}-D_{\text {mod }}}{D_{\text {ref }}}$

where $D_{\text {ref }}$ and $D_{\text {mod }}$ are, respectively, the drag of baseline and modification.

The compilation of the drag reduction characteristics and the nondimensional shock standoff distances $(\Delta)$ obtained at different jet pressure ratios and different flow control modes are shown in Table 4. This $\Delta$ is defined as the centerline distance from the blunt-body shock front to the leading edge of the blunt body divided by the blunt-body diameter. As seen in Table 4, it is observed that the $\Delta$ is 0.09 for the baseline (no flow field modification or injection), which is the minimum value compared with the other cases. For a single energy deposition region upstream of the blunt body (without injection), the $\Delta$ is 0.56 and the drag is reduced by $14.94 \%$. For a single opposing jet, the $\Delta=0.29$ at $P R=0.2$, whereas the $\Delta=0.22$ at $P R=0.4$, and there are $21.28 \%$ and $23.99 \%$ reductions in drag at $P R=0.2$ and 0.4 , respectively. For the combined method, the $\Delta=0.75$ at $P R=0.2$ and at $P R=0.4$, the $\Delta=0.61$, and the drag is reduced by $47.44 \%$ and $45.96 \%$

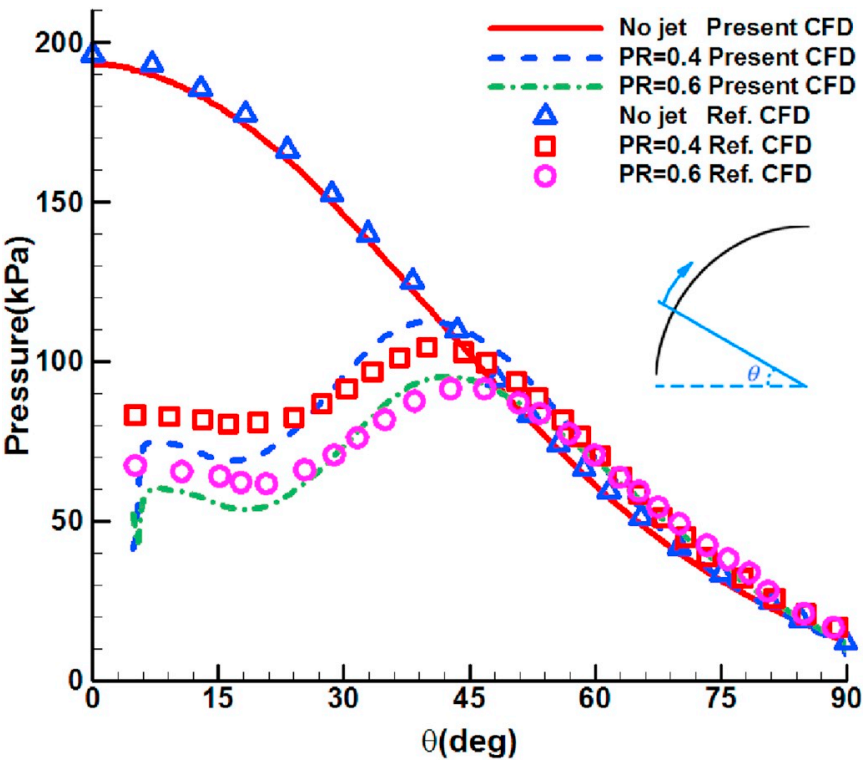

Fig. 5. Centerline surface pressure distributions at different pressure ratios.

Table 4

Comparison of representative quantities in different flow control modes.

\begin{tabular}{llll}
\hline & $\begin{array}{l}\text { Jet pressure } \\
\text { ratios }(P R)\end{array}$ & $\begin{array}{l}\text { Shock standoff } \\
\text { distance, } \Delta\end{array}$ & $\begin{array}{l}\text { Drag reduction } \\
\text { factor, } R D\end{array}$ \\
\hline Baseline & - & 0.09 & $0.00 \%$ \\
$\begin{array}{l}\text { Energy deposition } \\
\text { Opposing jet }\end{array}$ & - & 0.56 & $14.94 \%$ \\
Combined method & & 0.29 & $21.28 \%$ \\
Opposing jet & $P R=0.4$ & 0.75 & $47.44 \%$ \\
Combined method & & 0.22 & $23.99 \%$ \\
\hline
\end{tabular}

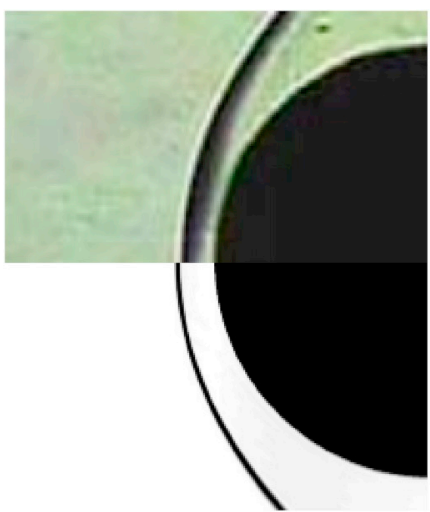

(a) No jet

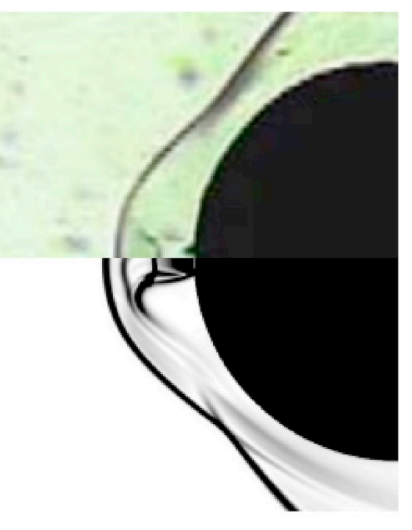

(b) $P R=0.4$

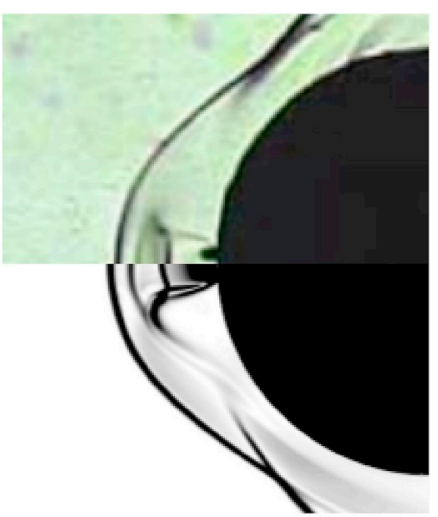

(c) $P R=0.6$

Fig. 4. Experimental Schlieren image (upper) versus calculated density gradient magnitude (lower). 


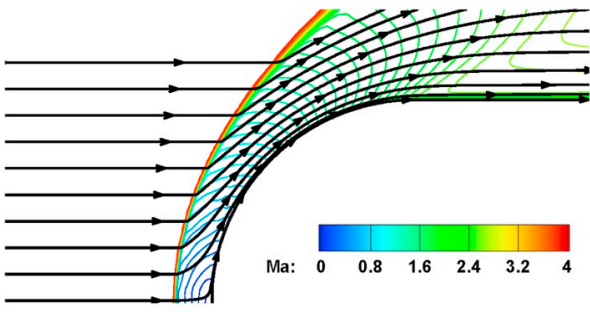

(a) Baseline

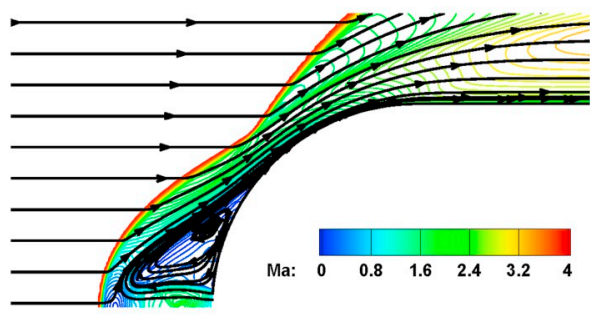

(c) Single opposing jet for $P R=0.2$

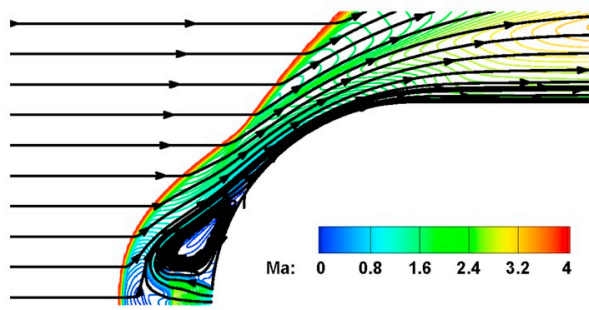

(e) Single opposing jet for $P R=0.4$

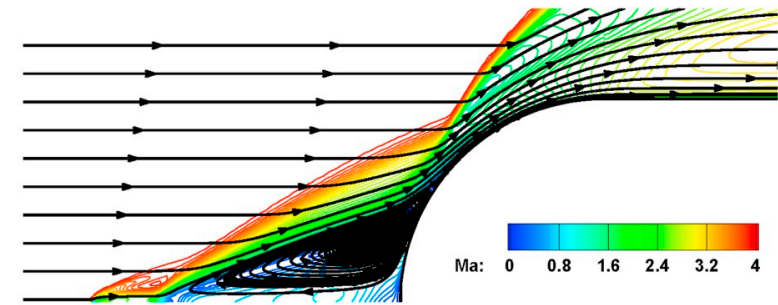

(b) Single energy deposition

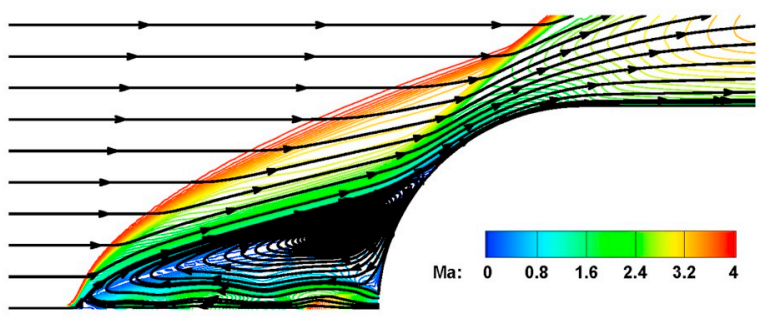

(d) Combined method for $P R=0.2$

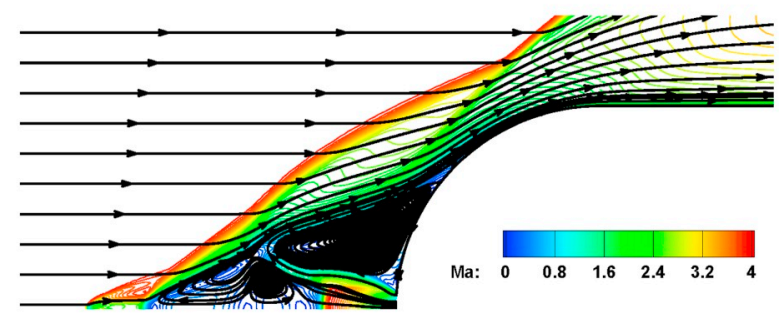

(f) Combined method for $P R=0.4$

Fig. 6. Mach number contours for different cases.

at $P R=0.2$ and 0.4 , respectively. It is noteworthy that the strategy of depositing an energized region of the upstream flow is shown to allow the minimum drag, especially for the long penetration mode (in the case of $P R=0.2$ ). This conclusion was also drawn by Khamooshi and Taylor et al. [20].

Line contours of the Mach number of the blunt body flow field generated for the different cases are depicted in Fig. 6. The bow shock is very thin and there is no flow separation as shown in Fig. 6(a). The streamlines overlapped on the Mach number contours clearly depict the separated flow caused by the upstream energy addition in Fig. 6(b). For the case of a single opposing jet, the recirculation region decreases, as PR increases from 0.2 to 0.4 as shown in Fig. 6(c) and (e). It is found that the recirculation region significantly increases when compared to the single opposing jet mode with the combined method, and two distinct recirculation regions exist in the case of the combined method with the $P R=0.4$, as depicted in Fig. 6(f).

The density gradient magnitudes at the nose of the blunt-body model in different flow control modes are illustrated in Fig. 7. In the case of the baseline, the bow shock is very close to the head of the blunt body, as seen in Fig. 7(a), the shock structures are recast by the control of the energy deposition, which induces shock interacts with the bow shock, as shown in Fig. 7(b). The flow structures of the single opposing jet ejected at the nose of the blunt model are depicted in Fig. 7(c) and (e). As the opposing jet emanates to the supersonic mainstream, the bow shock is pushed away from the head of the blunt body and the Mach disk is clearly recognized in the long penetration mode $(P R=0.2)$. The diamond-shaped pattern is formed by a series of compression and expansion shock waves ahead of the blunt body in the supersonic opposing flow. In the short penetration mode $(P R=0.4)$, the barrel shock is formed by compression waves reflected from the jet boundary in the opposing jet column. For the case of the combined method, the oblique shock is generated with the bow shock disappearing completely due to the upstream energy addition. The penetration of the opposing jet into mainstream increases and the shock standoff distance is obviously elongated, as shown in Fig. 7(d) and (f).

The convergence history of the long penetration mode $(P R=0.2)$ and the short penetration mode $(P R=0.4)$ are plotted in Figs. 8 and 9 , respectively. In addition, the convergence analysis for the single opposing jet and the combined method is conducted and presented. In the case of $P R=0.2$, the periodic oscillations of the residual and drag coefficient convergence histories are observed with the single the opposing jet. This instability of the opposing jet was observed both experimentally and numerically $[7,38]$. On the contrary, with the combined method, the residuals of continuity, velocity, and energy decline below $10^{-4}$ and the ranges of the variation of the drag coefficient do not exceed $1.0 \%$ after it reaches 2000 iterations and the steady-flow mode is met. Therefore, the upstream energy deposition is shown to significantly enhance the opposing jet stability. In the case of $P R=0.4$, the convergence history indicates that the flow field shows a stable state by the single opposing jet and combined method control technique.

The computed wall pressure distributions along the blunt body for all the cases are shown in Fig. 10. It is noticeable that the pressure value obtained by the control of the combined method has a significant reduction when compared with the single strategy of the opposing jet or energy deposition. For the combined methods, in the region from $5^{\circ}$ to $45^{\circ}$, the surface pressure in the case of $P R=0.2$ is lower than that in 


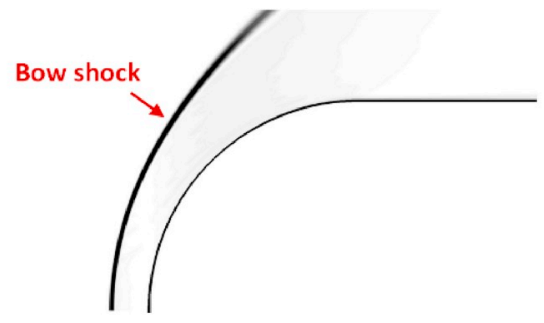

(a) Baseline

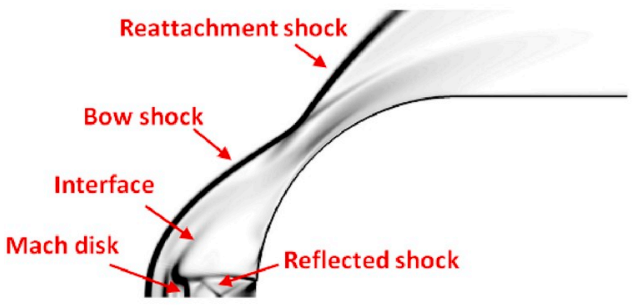

(c) Single opposing jet for $P R=0.2$

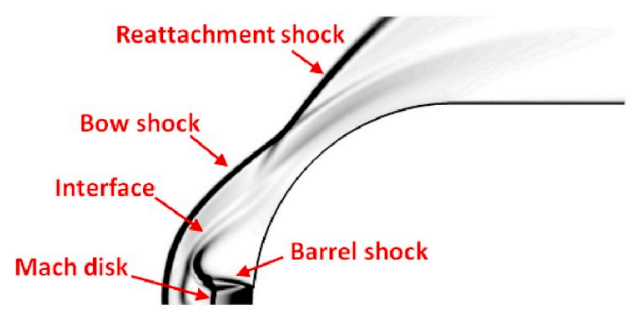

(e) Single opposing jet for $P R=0.4$

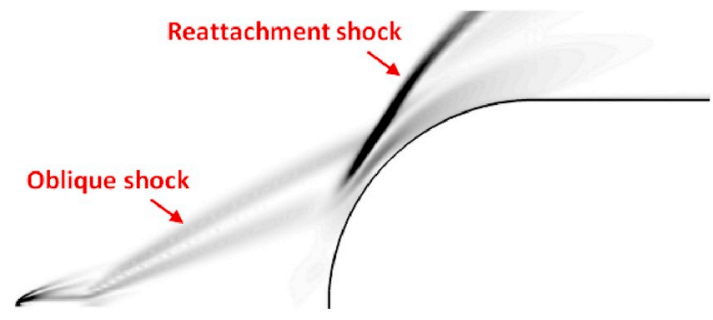

(b) Single energy deposition

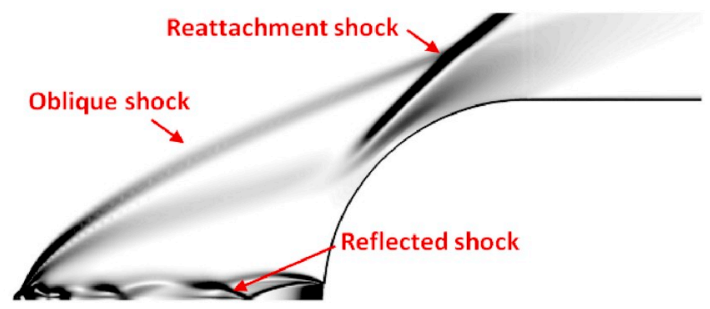

(d) Combined method for $P R=0.2$

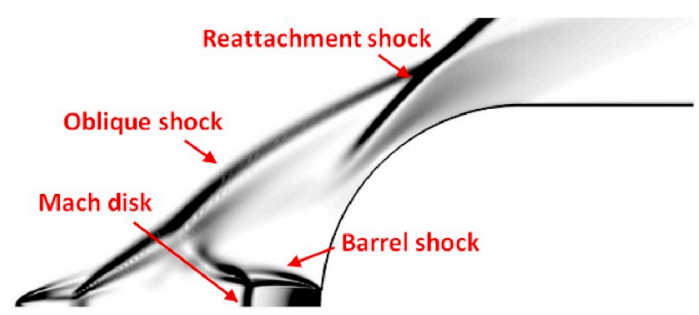

(f) Combined method for $P R=0.4$

Fig. 7. Density gradient magnitudes for different cases.

the case of $P R=0.4$. This implies that the depositing energy in the long penetration mode can reduce the drag characteristic more efficiently.

\subsection{Comparison of the power of energy deposition and jet injection}

In this research, the steady numerical approach is conducted. The energy deposition and jet injection are performed at constant rates [36] and the power for both should be provided and compared. The present heating effect employ via an energy addition rate with uniform distribution. Therefore, the power of energy deposition $Q$ given by Eq. (23), is defined as the product of cubic energy spot volume $V_{\mathrm{e}}$ and the energy deposition density $S_{\mathrm{e}}$.

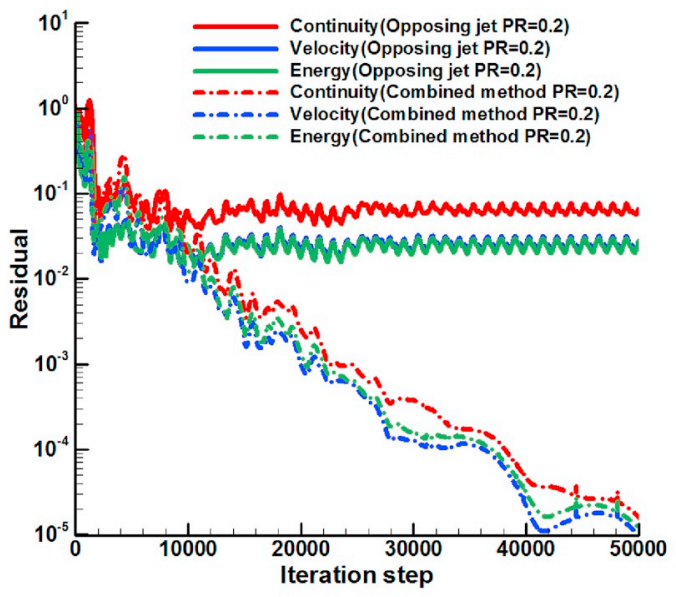

(a) Residuals convergence history.

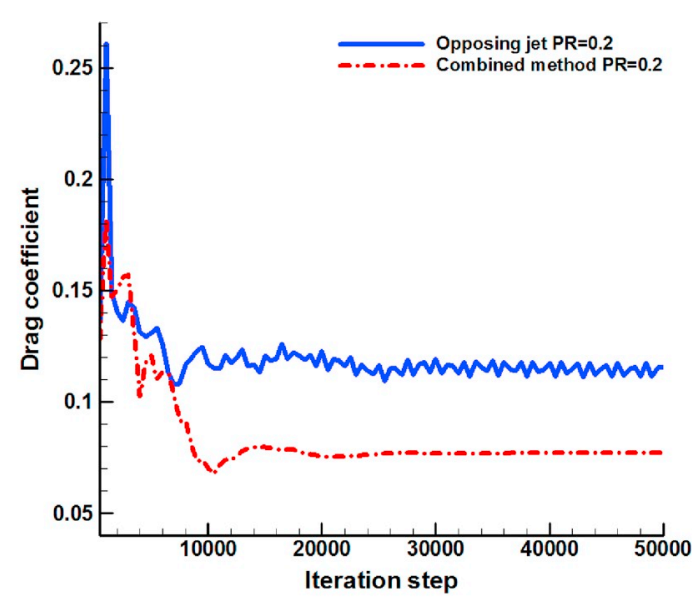

(b) Drag coefficient convergence history.

Fig. 8. Convergence history in case of $P R=0.2$. 


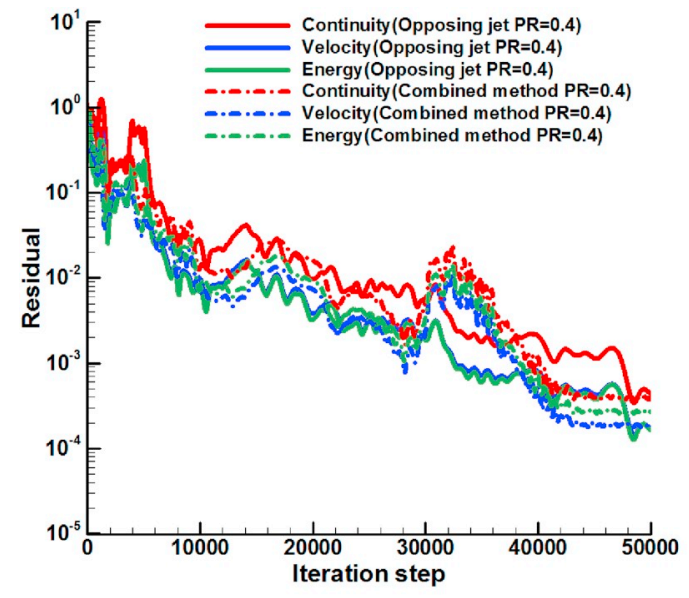

(a) Residuals convergence history.

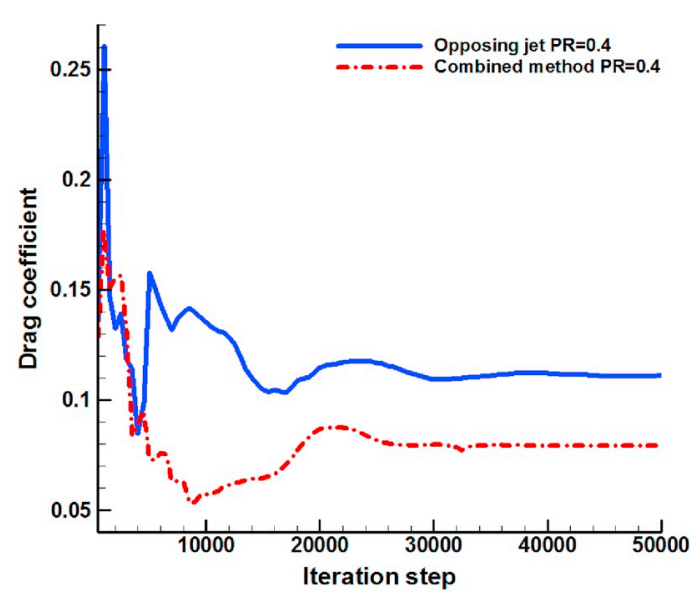

(b) Drag coefficient convergence history.

Fig. 9. Convergence history in case of $P R=0.4$.

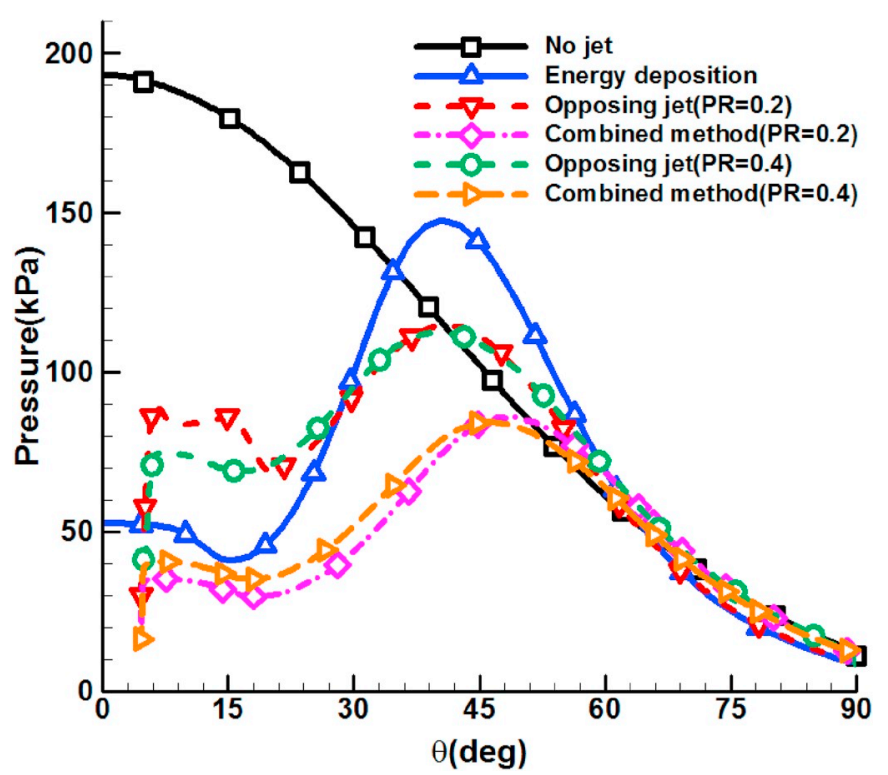

Fig. 10. Centerline surface pressure distributions at different control technique.

Table 5

The energy deposition information.

\begin{tabular}{llc}
\hline$S_{\mathrm{e}} / \mathrm{W} \cdot \mathrm{mm}^{-3}$ & $V_{\mathrm{e}} / \mathrm{mm}^{3}$ & $Q / \mathrm{W}$ \\
\hline 260 & 0.25 & 65 \\
\hline
\end{tabular}

Table 6

The jet injection information.

\begin{tabular}{lllll}
\hline & $\rho_{\mathrm{jet}} / \mathrm{kg} \cdot \mathrm{m}^{-3}$ & $v_{\mathrm{jet}} / \mathrm{m} \cdot \mathrm{s}^{-1}$ & $S_{\mathrm{jet}} / \mathrm{m}^{2}$ & $Q_{\mathrm{jet}} / \mathrm{W}$ \\
\hline$P R=0.2$ & 2.02 & 316.94 & 0.001256 & 80774.11 \\
$P R=0.4$ & 4.03 & 316.94 & 0.001256 & 161148.30 \\
\hline
\end{tabular}

$Q=S_{\mathrm{e}} \cdot V_{\mathrm{e}}$

As seen in Table 5, the energy deposition density $S_{\mathrm{e}}=260 \mathrm{~W} \mathrm{~mm}^{-3}$, the volume for energy spot $V_{\mathrm{e}}=0.25 \mathrm{~mm}^{3}$, and the power of energy deposition $Q=65 \mathrm{~W}$.

The power of jet injection $Q_{\text {jet }}$ is given by Eq. (24).
Table 7

Experimental design.

\begin{tabular}{lllllll}
\hline \multirow{2}{*}{ Test number } & \multicolumn{2}{l}{ Design variable } & & \multicolumn{2}{l}{ Drag characteristics } \\
\cline { 2 - 7 } & $D_{\mathrm{e}} / \mathrm{mm}$ & $L_{\mathrm{e}} / \mathrm{mm}$ & $S_{\mathrm{e}} / \mathrm{W} \cdot \mathrm{mm}^{-3}$ & $\mathrm{PR}$ & $\mathrm{RD}$ & $E_{\text {eff }}$ \\
\hline & & & & & \\
1 & 26.25 & 0.35 & 200 & 0.1 & 0.2365 & 959.74 \\
2 & 26.25 & 0.45 & 240 & 0.16 & 0.3118 & 496.03 \\
3 & 26.25 & 0.55 & 280 & 0.23 & 0.3836 & 286.49 \\
4 & 26.25 & 0.65 & 320 & 0.3 & 0.3640 & 144.10 \\
5 & 33.75 & 0.35 & 240 & 0.23 & 0.4659 & 1575.25 \\
6 & 33.75 & 0.45 & 200 & 0.3 & 0.4801 & 916.57 \\
7 & 33.75 & 0.55 & 320 & 0.1 & 0.3288 & 214.85 \\
8 & 33.75 & 0.65 & 280 & 0.16 & 0.4096 & 185.35 \\
9 & 41.25 & 0.35 & 280 & 0.3 & 0.5581 & 1617.34 \\
10 & 41.25 & 0.45 & 320 & 0.23 & 0.5254 & 626.85 \\
11 & 41.25 & 0.55 & 200 & 0.16 & 0.4652 & 486.38 \\
12 & 41.25 & 0.65 & 240 & 0.1 & 0.3945 & 208.26 \\
13 & 48.75 & 0.35 & 320 & 0.16 & 0.5069 & 1285.54 \\
14 & 48.75 & 0.45 & 280 & 0.1 & 0.4345 & 592.47 \\
15 & 48.75 & 0.55 & 240 & 0.3 & 0.6154 & 536.18 \\
16 & 48.75 & 0.65 & 200 & 0.23 & 0.5716 & 362.08 \\
\hline
\end{tabular}

$Q_{\text {jet }}=\left(\dot{m}_{\text {jet }} \cdot v_{\text {jet }}\right) \cdot v_{\text {jet }}=\left(\left(\rho_{\text {jet }} \cdot v_{\text {jet }} \cdot S_{\text {jet }}\right) \cdot v_{\text {jet }}\right) \cdot v_{\text {jet }}=\rho_{\text {jet }} \cdot v_{\text {jet }}^{3} \cdot S_{\text {jet }}$

where $\dot{m}_{\text {jet }}, v_{\text {jet }}, \rho_{\text {jet }}$, and $S_{\text {jet }}$ denote the jet mass flow rate, jet velocity, jet density, and jet orifice area, respectively.

Details of the jet injection information in different jet pressure ratios are tabulated in Table 6, it is noticeable that the power of jet injection $Q_{\text {jet }}$ is significantly greater than the power of energy deposition

\subsection{Parameters studies by variance analysis method}

The variance analysis technique coupled with the orthogonal experimental design was applied to investigate the parameter effects on drag reduction $[39,40]$. So far, the variance analysis method as a sensitivity analysis means has been widely employed to identify the influence of design variables on aerodynamic characteristics in the aerospace field [41].

In the current study, it is necessary to consider the energy consumption ratio and cost of the energy deposition in measuring drag reduction performance. The non-dimensional parameter of drag reduction effectiveness ( $E_{\text {eff }}$, given by Eq. (25), is defined as the ratio of the amount of profit gained by drag reduction to the amount spent for energy deposition [36,37]. 
Table 8

Results of between-subjects effects for $R D$.

\begin{tabular}{llllll}
\hline Source & Sum of squares & df & Mean square & F & Sig. \\
\hline$D_{\mathrm{e}}$ & $9.76 \mathrm{E}-02$ & 3 & $3.25 \mathrm{E}-02$ & 223.158 & 0.0005 \\
$L_{\mathrm{e}}$ & $3.95 \mathrm{E}-04$ & 3 & $1.32 \mathrm{E}-04$ & 0.903 & 0.5325 \\
$S_{\mathrm{e}}$ & $6.63 \mathrm{E}-04$ & 3 & $2.21 \mathrm{E}-04$ & 1.517 & 0.3702 \\
$P R$ & $5.98 \mathrm{E}-02$ & 3 & $1.99 \mathrm{E}-02$ & 136.764 & 0.0010 \\
Error & $4.37 \mathrm{E}-04$ & 3 & $1.46 \mathrm{E}-04$ & - & - \\
Total & $1.59 \mathrm{E}-01$ & 15 & - & - & - \\
\hline
\end{tabular}

Table 9

Results of between-subjects effects for $E_{\text {eff }}$

\begin{tabular}{llllll}
\hline Source & Sum of squares & df & Mean square & F & Sig. \\
\hline$D_{\mathrm{e}}$ & $1.85 \mathrm{E}+05$ & 3 & $6.15 \mathrm{E}+04$ & 5.2796 & 0.1026 \\
$L_{\mathrm{e}}$ & $3.03 \mathrm{E}+06$ & 3 & $1.01 \mathrm{E}+06$ & 86.5435 & 0.0021 \\
$S_{\mathrm{e}}$ & $4.37 \mathrm{E}+04$ & 3 & $1.46 \mathrm{E}+04$ & 1.2487 & 0.4297 \\
$P R$ & $2.12 \mathrm{E}+05$ & 3 & $7.08 \mathrm{E}+04$ & 6.0763 & 0.0863 \\
Error & $3.50 \mathrm{E}+04$ & 3 & $1.17 \mathrm{E}+04$ & - & - \\
Total & $3.50 \mathrm{E}+06$ & 15 & - & - & - \\
\hline
\end{tabular}

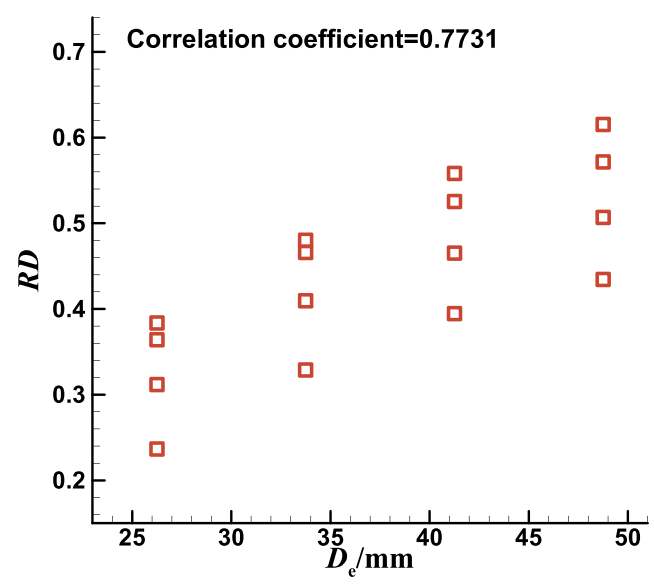

(a) De versus $R D$

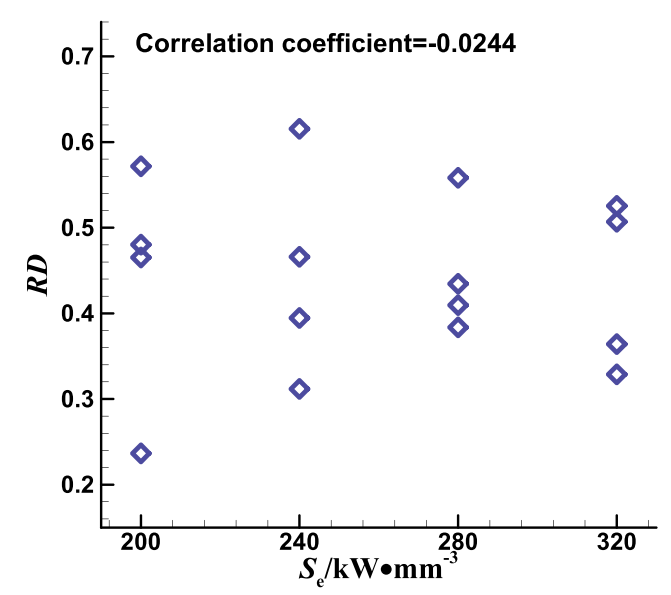

(c) $S_{\mathrm{e}}$ versus $R D$
$E_{\text {eff }}=\frac{\left(D_{\text {ref }}-D_{\text {mod }}\right) V_{\infty}}{Q}$

where $V_{\infty}$ and $Q$ denote the velocity and the power of energy deposition, respectively.

For the combinatorial configuration of the upstream energy deposition and opposing jet, four design variances are considered, namely, $D_{\mathrm{e}}, L_{\mathrm{e}}, S_{\mathrm{e}}$ and $P R$. Each design variable has four levels, i.e. $D_{\mathrm{e}} \varepsilon$ $\{26.25,33.75,41.25,48.75\}, L_{\mathrm{e}} \varepsilon\{0.35,0.45,0.55,0.65\}, S_{\mathrm{e}} \varepsilon\{200$, $240,280,320\}, P R \varepsilon\{0.1,0.16,0.23,0.3\}$, and the other design variables are set as a constant.

The orthogonal experimental design table $L_{16}\left(4^{5}\right)$ is used for the design variable arrangement(see Table 7). The first four columns of the table are allotted to the design parameters and random errors are arranged in the last column $[26,41]$.

Table 8 and Table 9 show the results of between-subjects effects for drag reduction factor $(R D)$ and drag reduction effectiveness $\left(E_{\text {eff }}\right)$, respectively. The term ' $\mathrm{df}$ ' denotes the freedom degree, the term ' $\mathrm{F}$ ' is the statistic prescribed as the mean square ratio of the factor to the error term, and the term 'Sig.' is the statistical indication applied to estimate the possibility of the result occurrence. The confidence interval is separated by 0.05 in the process of variance analysis.

In Table 8 , it is observed that, for drag reduction factor $(R D)$, the values of Sig. of the distance between the blunt body and the energy

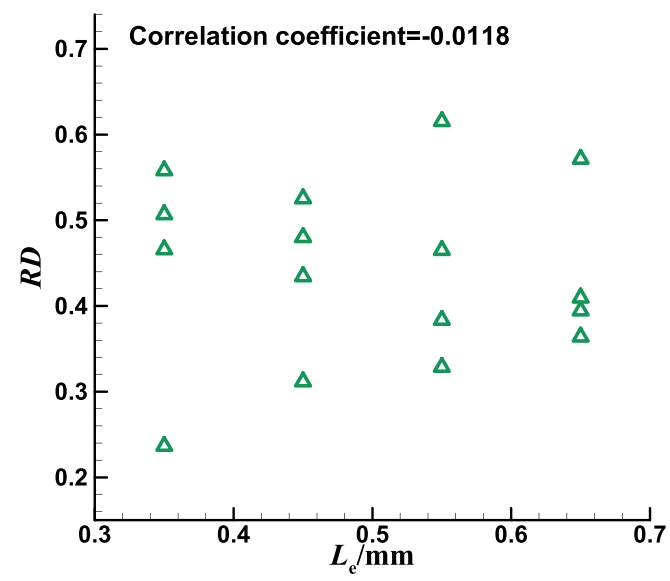

(b) $L_{\mathrm{e}}$ versus $R D$

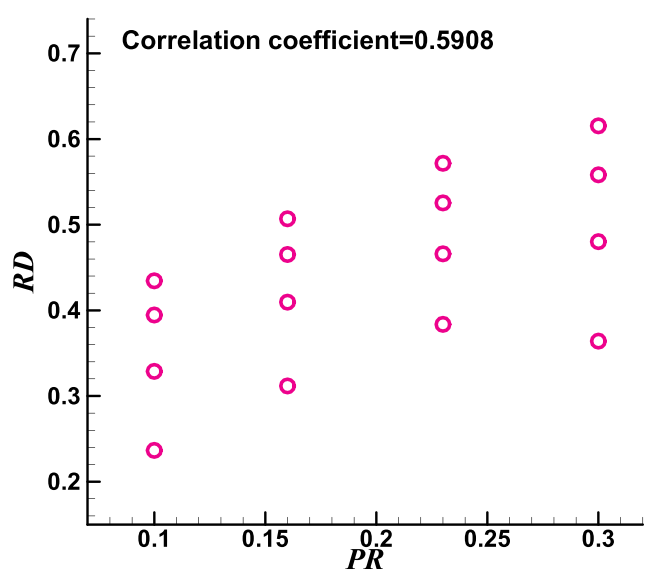

(d) PR versus $R D$

Fig. 11. Correlation plots demonstrating the influence on $R D$. 


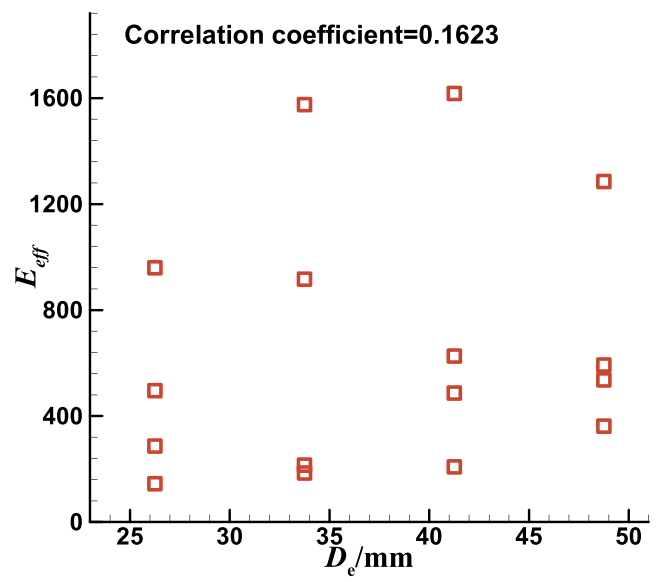

(a) $D_{\text {e versus }} E_{\text {eff }}$

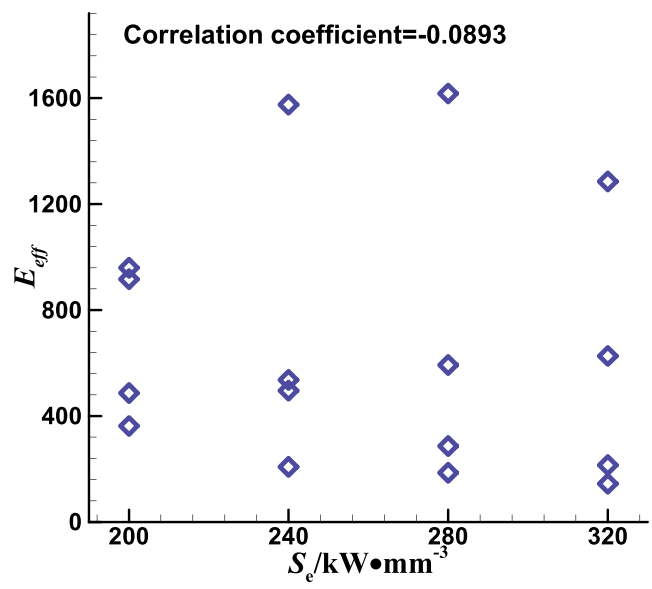

(c) $S_{\text {e versus }} E_{\text {eff }}$

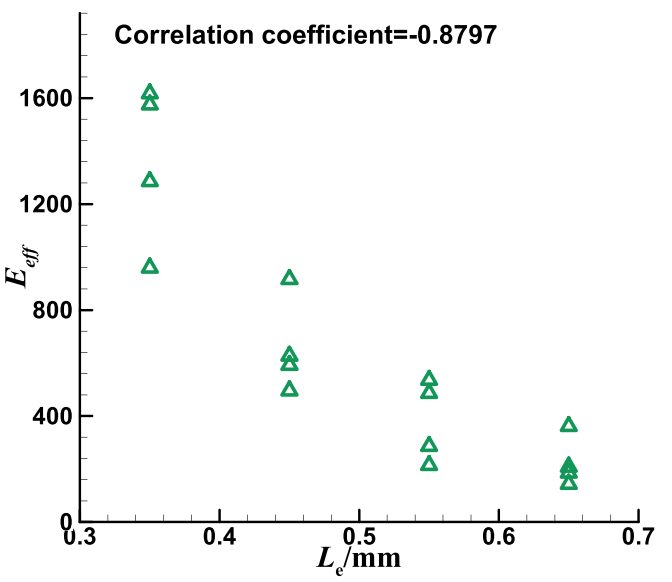

（b） $L_{\mathrm{e}}$ versus $\boldsymbol{E}_{\text {eff }}$

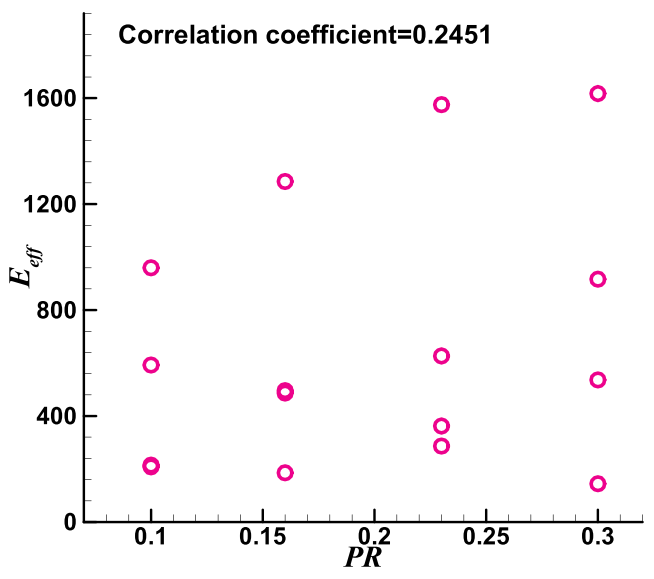

(d) $P R$ versus $E_{\text {eff }}$

Fig. 12. Correlation plots demonstrating the influence on $E_{\text {eff. }}$.

spot $\left(D_{\mathrm{e}}\right)$, and the jet-to-freestream total-pressure ratio $(P R)$ are less than 0.05. This implies that the effects of $D_{\mathrm{e}}$ and $P R$ on the drag reduction factor are substantial. However, the effects of the other two design variables, namely, $L_{\mathrm{e}}$ and $S_{\mathrm{e}}$, are not obvious. The values of the distance between the blunt body and energy spot $\left(D_{\mathrm{e}}\right)$ and the jet-tofreestream total-pressure ratio $(P R)$ must be investigated first in the drag reduction design using the combinational means of the upstream energy deposition and opposing jet.

The results of between-subjects effects for drag reduction effectiveness $\left(E_{\text {eff }}\right)$ are shown in Table 9. It is observed that for drag reduction effectiveness $E_{\text {eff, }}$ only the Sig. of the side length of the energy deposition cube $\left(L_{\mathrm{e}}\right)$ is less than 0.05 . This implies that the effects of $L_{\mathrm{e}}$ on the drag reduction effectiveness are substantial, but the influences of the other three design variables, namely $D_{\mathrm{e}}, S_{\mathrm{e}}$, and $P R$ are not substantial. Therefore, the side length of the energy deposition cube $\left(L_{\mathrm{e}}\right)$ is more important for drag reduction efficiency.

Furthermore, the linear regression method is used to verify the results of the variance analysis [42,43]. The correlation coefficient is calculated by linear regression, thereby indicating the linear relationship between the design variables and the results. The closer the correlation coefficients are to 1 or -1 , the stronger the correlation is. Scatter plots along with the correlation coefficients are shown in Fig. 11 and Fig. 12. It is observed that, for the drag reduction factor $(R D)$, the correlation coefficients of $D_{\mathrm{e}}$ and $P R$ are 0.7731 and 0.5908 , respectively. These values are much larger than the other two design variables, namely $L_{\mathrm{e}}$ and $S_{\mathrm{e}}$. As seen in Fig. 12, the design variable of $L_{\mathrm{e}}$ has a significant impact on drag reduction effectiveness. This conclusion is consistent with that obtained by variance analysis method. Moreover, the drag reduction factor $(R D)$ increases with the design variable of increasing $P R$ or $D_{\mathrm{e}}$, and the drag reduction effectiveness $\left(E_{\text {eff }}\right)$ decreases with the design variable of increasing $L_{\mathrm{e}}$.

\section{Conclusion}

In this paper, the comparisons of the flow field and drag characteristics upon the blunt-body model with the energy deposition only, the opposing jet only, as well as, the combinational configuration of the upstream energy deposition and opposing jet are discussed. The parameter effects of the combined control mode are demonstrated and analyzed by the variance analysis method coupled with the orthogonal experimental design. Main findings are summarized as follow:

(1) The superiority of the opposing jet coupled with the upstream energy deposition in the blunt-body drag reduction is clearly seen by the results. The drag characteristics are greatly reduced by the combinational configuration of the control than the single strategies 
of the opposing jet and the energy deposition. Moreover, the maximum overall drag reduction happens in the long penetration mode.

(2) The flow field of the single opposing jet of long penetration mode presents the periodic oscillation. The upstream energy deposition can enhance significantly the stability of the opposing jet in the long penetration mode.

(3) In the drag reduction design of the blunt body with the combinational configuration of the upstream energy deposition and the opposing jet, both of the value of the distance between the blunt body and energy spot $\left(D_{\mathrm{e}}\right)$ and the jet-to-freestream total-pressure ratio $(P R)$ should be considered first since small variance may lead to a substantial effect of the drag reduction factor $(R D)$, whereas the design variable of $L_{\mathrm{e}}$ has a significant influence on the drag reduction effectiveness $\left(E_{\text {eff }}\right)$. Furthermore, the combined control mode with the longer distance of the energy spot and a bigger jetto-freestream total-pressure ratio would contribute to the drag reduction. The energy spot with a smaller side length shows better drag reduction efficiency.

The thermal protection is another important process during the design of the supersonic/hypersonic vehicles, but the drag and heat release reductions on vehicles are often conflicting [19], and they should be solved by using a multi-objective design optimization approach $[44,45]$ in the future study.

\section{Acknowledgments}

The authors are very grateful to Professor Chao Yan for the valuable discussions from Beihang University. This work was supported by the Postdoctoral Project for Advanced Engineering Construction of the Chinese Academy of Sciences.

\section{References}

[1] W. Huang, L. Ma, Z.G. Wang, M. Pourkashanian, D.B. Ingham, S.B. Luo, J. Lei, A parametric study on the aerodynamic characteristics of a hypersonic waverider vehicle, Acta Astronaut. 69 (2011) 135-140.

[2] J. Guo, G. Lin, X. Bu, H. Li, Parametric study on the heat transfer of a blunt body with counterflowing jets in hypersonic flows, Int. J. Heat Mass Tran. 121 (2018) 84-96.

[3] R. Zhang, W. Huang, Y. Li, C. Zheng, R. Moradic, Drag and heat flux reduction induced by the pulsed counterflowing jet with different waveforms on a blunt body in supersonic flows, Acta Astronaut. 160 (2019) 635-645.

[4] J. Gopalan, V. Menezes, K. Reddy, T. Hashimoto, M. Sun, K. Takayama. Hypersonic buzz phenomenon on the spiked blunt cones, AIAA 41st Aerospace Sciences Meetings. 2003 Jan 6-9; Reno, USA. Reston.

[5] V. Menezes, S. Saravanan, G. Jagadeesh, K.P. Reddy, Experimental investigations of hypersonic flow over highly blunted cones with aerospikes, AIAA J. 41 (10) (2003) 1955-1966.

[6] P.J. Finley, The flow of a jet from a body opposing a supersonic free stream, J. Fluid Mech. 26 (2) (1966) 337-368.

[7] K. Hayashi, S. Aso, Y. Tani, Experimental study on thermal protection system by opposing jet in supersonic flow, J. Spacecraft Rockets 43 (2006) 233-236.

[8] L.W. Chen, G.L. Wang, X.Y. Lu, Numerical investigation of a jet from a blunt body opposing a supersonic flow, J. Fluid Mech. 684 (2011) 85-110.

[9] W. Huang, J. Liu, Z.X. Xia, Drag reduction mechanism induced by a combinational opposing jet and spike concept in supersonic flows, Acta Astronaut. 115 (2015) 24-31.

[10] Z. Eghlima, K. Mansour, K. Fardipour, Heat transfer reduction using combination of spike and counterflow jet on blunt body at high Mach number flow, Acta Astronaut. 143 (2018) 92-104.

[11] Y.F. Liu, Z.L. Jiang, Concept of non-ablative thermal protection system for hypersonic vehicles, AIAA J. 51 (3) (2013) 584-590.

[12] W. Huang, L. Yan, J. Liu, L. Jin, J. Tan, Drag and heat reduction mechanism in the combinational opposing jet and acoustic cavity concept for hypersonic vehicles, Aero. Sci. Technol. 42 (2015) 407-414.

[13] X. Sun, W. Huang, Z. Guo, L. Yan, Multiobjective design optimization of hypersonic combinational novel cavity and opposing jet concept, J. Spacecraft Rockets 54 (3) (2017) 1-10.

[14] X. Sun, Z. Guo, W. Huang, S. Li, L. Yan, A study of performance parameters on drag and heat flux reduction efficiency of combinational novel cavity and opposing jet concept in hypersonic flows, Acta Astronaut. 131 (2017) 204-225.
[15] H. Lu, W. Liu, Thermal protection efficiency of forward-facing cavity and opposing jet combinational configuration, J. Therm. Sci. 21 (4) (2012) 342-347.

[16] Z. Wang, X. Sun, W. Huang, S. Li, L. Yan, Experimental investigation on drag and heat flux reduction in supersonic/hypersonic flows: a survey, Acta Astronaut. 129 (2016) 95-110.

[17] W. Huang, Z. Chen, L. Yan, B. Yan, Z. Du, Drag and heat flux reduction mechanism induced by the spike and its combinations in supersonic flows: a review, Prog. Aero. Sci. 105 (2019) 31-39.

[18] X. Sun, W. Huang, M. Ou, R. Zhang, S. Li, A survey on numerical simulations of drag and heat reduction mechanism in supersonic/hypersonic flows, Chin. J. Aeronaut. 32 (4) (2019) 771-784.

[19] W. Huang, A survey of drag and heat reduction in supersonic flows by a counterflowing jet and its combinations, J. Zhejiang Univ. - Sci. 16 (7) (2015) 551-561.

[20] A. Khamooshi, T. Taylor, D.W. Riggins, Drag and heat transfer reductions in highspeed flows, AIAA J. 45 (10) (2007) 2401-2413.

[21] C.D. Marley, D.W. Riggins, Numerical study of novel drag reduction techniques for hypersonic blunt bodies, AIAA J. 49 (9) (2011) 1871-1882.

[22] D. Das, S. Desai, V. Kulkarni, H. Gadgil, Performance assessment of energy deposition based drag reduction technique for Earth and Mars flight conditions, Acta Astronaut. 159 (2019) 418-428.

[23] B. Launder, D.B. Spalding, The numerical computation of turbulent flows, Comput. Methods Appl. Mech. Eng. 3 (2) (1974) 269-289.

[24] L.K. Sherrie, R.T. Biedron, C.L. Rumsey, CFL3D User's Manual (Version 5.0), (1998) NASA/TM-1998-208444.

[25] S. Ju, C. Yan, X. Wang, Y. Qin, Z. Ye, Optimization design of energy deposition on single expansion ramp nozzle, Acta Astronaut. 140 (2017) 351-361.

[26] S. Ju, C. Yan, X. Wang, Y. Qin, Z. Ye, Effect of energy addition parameters upon scramjet nozzle performances based on the variance analysis method, Aero. Sci. Technol. 70 (2017) 511-519.

[27] X. Sun, W. Huang, Z. Guo, S. Li, L. Yan, Drag and heat reduction mechanism induced by a combinational novel cavity and counterflowing jet concept in hypersonic flows, Acta Astronaut. 126 (2016) 109-119.

[28] M. Ou, L. Yan, W. Huang, S. Li, L. Li, Detailed parametric investigations on drag and heat flux reduction induced by a combinational spike and opposing jet concept in hypersonic flows, Int. J. Heat Mass Tran. 126 (2018) 10-31.

[29] W. Huang, W. Liu, S. Li, Z. Xia, J. Liu, Z. Wang, Influences of the turbulence model and the slot width on the transverse slot injection flow field in supersonic flows, Acta Astronaut. 73 (2012) 1-9.

[30] R. Zhang, W. Huang, L. Yan, L. Li, S. Li, R. Moradi, Numerical investigation of drag and heat flux reduction mechanism of the pulsed counterflowing jet on a blunt body in supersonic flows, Acta Astronaut. 146 (2018) 123-133.

[31] F.R. Menter, Two-equation eddy-viscosity turbulence models for engineering applications, AIAA J. 32 (8) (1994) 1598-1605.

[32] J. Blazek, Computational Fluid Dynamics: Principles and Applications, second ed., Elsevier Ltd., Ox-ford, 2005.

[33] S. Ju, C. Yan, X. Wang, Y. Qin, Z. Ye, Sensitivity analysis of geometric parameters upon the aerothermodynamic performances of Mars entry vehicle, Int. J. Heat Mass Tran. 120 (2018) 597-607.

[34] N.N. Smirnov, V.B. Betelin, V.F. Nikitin, L.I. Stamov, D.I. Altoukhov, Accumulation of errors in numerical simulations of chemically reacting gas dynamics, Acta Astronaut. 117 (2015) 338-355.

[35] N.N. Smirnov, V.B. Betelin, R.M. Shagaliev, V.F. Nikitin, I.M. Belyakov, Yu.N. Deryuguin, S.V. Aksenov, D.A. Korchazhkin, Hydrogen Fuel Rocket Engines Simulation Using LOGOS Code vol. 39, (2014), pp. 10748-10756.

[36] G.M. Ashwin, J. Bibin, Concentrated energy addition for active drag reduction in hypersonic flow regime, Acta Astronaut. 142 (2018) 221-231.

[37] D. Siddesh, K. Vinayak, G. Hrishikesh, J. Bibin, Aerothermodynamic considerations for energy deposition based drag reduction technique, Appl. Therm. Eng. 122 (2017) 451-460.

[38] A. Bibi, A. Maqsood, S. Sherbaz, L. Dala, Drag reduction of supersonic blunt bodies using opposing jet and nozzle geometric variations, Aero. Sci. Technol. 69 (2017) 244-256.

[39] W. Huang, M. Pourkashanian, L. Ma, D.B. Ingham, S. Luo, Z. Wang, Effect of geometric parameters on the drag of the cavity flameholder based on the variance analysis method, Aero. Sci. Technol. 21 (2012) 24-30.

[40] W. Huang, Z. Wang, D.B. Ingham, L. Ma, M. Pourkashanian, Design exploration for a single expansion ramp nozzle (SERN) using data mining, Acta Astronaut. 83 (2013) 10-17.

[41] S. Ju, C. Yan, Z. Ye, Multi-parametric sensitivity analysis of air breathing hypersonic vehicle, J. Beijing Univ. Aeronaut. Astronaut. 43 (5) (2017) 927-935 (in Chinese).

[42] G. Ciulla, A. D'Amico, Building energy performance forecasting: a multiple linear regression approach, Appl. Energy 253 (2019) 113500.

[43] B.R. Bettis, S. Hosder, Uncertainty quantification in hypersonic reentry flows due to aleatory and epistemic uncertainties, $49^{\text {th }}$ AIAA Aerospace Sciences Meeting Including the New Horizons Forum and Aerospace Exposition. Orlando, Florida, 2011.

[44] M. Ou, L. Yan, W. Huang, T. Zhang, Design exploration of combinational spike and opposing jet concept in hypersonic flows based on CFD calculation and surrogate model, Acta Astronaut. 155 (2019) 287-301.

[45] W. Huang, Design exploration of three-dimensional transverse jet in a supersonic crossflow based on data mining and multi-objective design optimization approaches, Int. J. Hydrogen Energy 39 (8) (2014) 3914-3925. 